\title{
Médiévales
}

Langues, Textes, Histoire

72 | printemps 2017

Roman du Genji et société aristocratique au Japon

\section{Le ministre Fujiwara no Michinaga (966-1027) : politique matrimoniale, apparat et liens de clientèle à l'époque du Roman du Genji}

The Minister Fujiwara no Michinaga (966-1027): Matrimonial Politics, Pageantry and Patronage at the Time of the Tale of Genji.

Charlotte von Verschuer

\section{OpenEdition}

Journals

\section{Édition électronique}

URL : https://journals.openedition.org/medievales/8024

DOI : 10.4000/medievales.8024

ISSN : 1777-5892

\section{Éditeur}

Presses universitaires de Vincennes

\section{Édition imprimée}

Date de publication : 22 juin 2017

Pagination : 21-38

ISBN : 978-2-84292-612-0

ISSN : 0751-2708

\section{Référence électronique}

Charlotte von Verschuer, « Le ministre Fujiwara no Michinaga (966-1027) : politique matrimoniale, apparat et liens de clientèle à l'époque du Roman du Genji », Médiévales [En ligne], 72 I printemps 2017, mis en ligne le 28 février 2019, consulté le 22 avril 2022. URL : http://journals.openedition.org/ medievales/8024; DOI : https://doi.org/10.4000/medievales.8024 


\section{Le ministre Fujiwara no Michinaga (966-1027) : politique matrimoniale, apparat et liens de clientèle à l'époque du Roman du Genji}

L'époque du Roman $d u$ Genji connut une mutation dans les méthodes de gouvernement. La cour impériale, siège de l'administration publique, était alors dominée par le ministre Fujiwara no Michinaga. Ce personnage fut à l'origine d'un changement des mécanismes du pouvoir, précipitant la transformation, déjà commencée depuis au moins un siècle, du système bureaucratique fondé au début du VIII ${ }^{\mathrm{e}}$ siècle en un système clientéliste. Pour atteindre ses fins politiques, Michinaga mit à contribution la politique matrimoniale et son réseau de clientèle. En outre, sa mise en scène de cérémonies fastueuses contribua à exalter son prestige à la cour. L'exclamation suivante traduit parfaitement l'ambition du ministre :

Ah ! Je puis penser que cet âge est vraiment le mien, quand de la lune rien ne vient diminuer la plénitude ${ }^{1}$.

Quand Fujiwara no Michinaga (966-1027) composa ce poème, au dixième mois de l'année 1018, lors du banquet officiel donné à l'occasion du mariage de sa fille Ishi (999-1036) avec l'empereur Goichijô (1008-1036, r. 10161036) $)^{2}$, il se trouvait au sommet de sa gloire. Il était lui-même à l'origine de

1. Shôyûki (Notes journalières de Fujiwara no Sanesuke [957-1046]), TAKEI K. éd., Kannin 2.10.16 (1018), Kyôto, 1968. Midô kanpakuki zenchûshaku, YAMANAKA Y. éd., $1^{\text {re }}$ publication en 5 vol., Kyôto, 1985-1997, $2^{\text {e }}$ publication en 14 vol., Kyôto, 20032012 ; trad. F. HÉRAIL, Notes journalières de Fujiwara no Michinaga, ministre à la cour de Heian (995-1018) : traduction du Midô kanpakuki, Genève, 3 vol. , 1987-1988-1991 (ici vol. 3, p. 544). Michinaga est mort le quatrième jour du douzième mois qui, stricto sensu, correspond au 3 janvier 1028, mais nous donnons ici la date de 1027 pour commodité, car tous les dictionnaires japonais disent 1027.

2. Midô kanpakuki zenchûshaku, Kannin 2.10.16. (1018), trad. F. HÉRAIL, Notes journalières..., vol. 3, p. 540-544. 
cette union qu'il avait arrangée en dépit de la différence d'âge des époux et de leurs liens de parenté.

Lorsque fut rédigé le Roman du Genji (Genji monogatari), Fujiwara no Michinaga était le personnage le plus puissant de la cour impériale. Descendant d'une illustre famille dont l'histoire est étroitement liée à celle de l'État japonais ancien, dit État régi par les codes, il en a porté le prestige à son apogée ${ }^{3}$. Les Fujiwara avaient assuré leur puissance grâce à une politique consistant à placer leurs filles au palais comme épouses impériales, ce qui leur permettait d'exercer les fonctions de régent auprès de l'empereur régnant et de contrôler la succession impériale ainsi que le système de promotion des fonctionnaires de cour. Mais Michinaga a porté cette prééminence à un degré sans précédent. Sa puissance et son faste ont frappé les esprits et il est resté célèbre sous le nom de «Chancelier de la Chapelle » (Midô kanpaku), bien qu'il n'ait réellement occupé la fonction de chancelier que pendant quelques mois. Ce surnom fait référence au temple Hôjôji qu'il avait fait bâtir à côté de sa résidence et où il a fini ses jours. Grâce à son appartenance à la famille Fujiwara d'où venaient traditionnellement les hauts dignitaires du gouvernement, Michinaga occupa pendant la plus grande partie de sa carrière le poste de ministre de Gauche (sadaijin), ce qui faisait de lui le chef du Conseil d'État. Il s'est fait accorder par ordre impérial le privilège de procéder à un «examen officieux» (nairan) de tous les documents publiés par l'empereur ou adressés par lui, ce qui lui donnait la haute main sur l'ensemble de l'administration ${ }^{4}$. Il a su également s'entourer d'une nombreuse clientèle qui lui a permis d'accumuler une immense fortune tout au long de sa vie. Michinaga a par ailleurs eu la chance d'avoir une nombreuse descendance. Ses deux épouses lui ont donné douze enfants, qui sont devenus des dignitaires de la cour ou des épouses impériales. Il a ainsi fait nommer son fils Fujiwara no Yorimichi (992-1074) régent et tuteur de plusieurs empereurs successifs, tout en étant lui-même le mentor des souverains qui étaient ses gendres. Même après son

3. La famille Fujiwara tire son origine de l'antique famille Nakatomi. Celle-ci avait d'abord exercé des fonctions religieuses avant de se retrouver au centre du pouvoir grâce à Nakatomi no Kamatari (614-669), qui, dans l'entourage de l'empereur Tenji (626-668), joua un rôle essentiel dans l'adoption par le Japon d'un système bureaucratique imité de celui de la Chine, ainsi qu'au fils de celui-ci Nakatomi no Fuhito (659-720), ministre de l'impératrice Jitô (645-703) et l'un des artisans de l'État régi par les codes, qui prit le nom de Fujiwara. Les quatre fils de Fuhito, tous disparus au cours de l'année 737 dans une épidémie de variole, furent les fondateurs de quatre branches de la famille, dont la branche du Nord, qui parvint à asseoir son pouvoir à partir du milieu du IX ${ }^{\mathrm{e}}$ siècle.

4. C'est en principe le régent qui était chargé de procéder à cet « examen officieux » mais, du temps de Michinaga, il n'y a pas eu de régent de 995 à 1016. Cet « examen officieux » permettait de contrôler les textes adressés à l'empereur ou émis par lui, de les faire rectifier et d'infléchir certaines décisions. Sur cette question, voir ÔTSU T., Michinaga to kyûtei shakai, Tôkyô, 2001. 
entrée en religion, en 1019, Michinaga a continué à diriger les affaires de l'État, et ce jusqu'à sa mort survenue en 1027. La lignée du «Chancelier de la Chapelle » s'est perpétuée bien au-delà de sa disparition. Ses descendants en ligne directe ont en effet assumé la dignité impériale jusqu'en 1072, soit durant près d'un demi-siècle.

Comme beaucoup de hauts dignitaires de l'époque de Heian (7941185), Michinaga rédigeait des notes journalières. Le manuscrit autographe du journal du «Chancelier de la Chapelle » et une copie du $\mathrm{XI}^{\mathrm{e}}$ siècle de ce document sont conservés dans les archives Yômei Bunko de Kyôto et classés « trésor national » par le gouvernement japonais. Ils ont été traduits, annotés et commentés par Francine Hérail dans un ouvrage en trois volumes intitulé Notes journalières de Fujiwara no Michinaga, qui sera plusieurs fois mentionné dans la suite de notre étude. Cette œuvre, où les affaires de famille et les cérémonies occupent une place plus importante que les questions politiques, permet de suivre la vie de son auteur pratiquement au jour le jour, notamment entre les années 998 et 1021. Dans le présent article, nous retraçons les aspects de la carrière du grand chancelier qui ont le plus contribué à sa réussite et à sa gloire de son vivant et même au-delà, à savoir sa politique matrimoniale et l'immense fortune qu'il a accumulée. Dans un second temps nous nous intéresserons plus particulièrement aux cérémonies organisées par lui, ainsi qu'au faste dont il s'entourait.

\section{Une politique matrimoniale couronnée de succès}

La célébration du mariage de sa fille Ishi en 1018 marqua le plus beau jour de la vie de Fujiwara no Michinaga. Il avait en effet réussi à ce que trois de ses filles deviennent impératrices. «Le Grand miroir » (Ôkagami), compilé par un auteur anonyme au XII ${ }^{\mathrm{e}}$ siècle, relate les faits en ces termes :

La première fille de Michinaga, Shôshi, fut donnée en épouse à l'empereur Ichijô, à l'âge de douze ans. En l'an mil, alors qu'elle était princesse, elle fut élevée au rang d'impératrice épouse ; puis elle donna naissance à deux princes [Atsuhira et Atsunaga] qui devinrent bientôt empereur et prince héritier [...]. La deuxième fille, Kenshi, directrice du Service intérieur du palais, fut mariée au prince héritier, le futur empereur Sanjô, et devint impératrice épouse à l'âge de dix-neuf ans. L'année suivante, en 1013, elle mit au monde une princesse [Teishi] [...]. La troisième fille de Michinaga, Ishi, elle aussi directrice du Service intérieur, épousa en 1018 l'empereur [Goichijô], peu de temps après sa vêture du pantalon ${ }^{5}$, à l'âge de onze ans. Elle est l'impératrice en titre et vit au palais. La quatrième fille, Kishi,

5. Cérémonie qui marque le passage de la toute petite enfance à l'enfance. 
également directrice du Service intérieur, est devenue l'épouse du prince héritier [Atsunaga] à quinze ans, alors que celui-ci en avait treize [...]. Nul autre dignitaire de notre pays n'a vu trois de ses filles être épouses impériales. La maison de Michinaga a été, à elle seule, gratifiée de trois impératrices toutes contemporaines, l'une, archi-douairière [Shôshi], l'autre, douairière [Kenshi] et la troisième, épouse impériale [Ishi]. C'est une rare bénédiction ${ }^{6}$.

Durant toute sa carrière, Fujiwara no Michinaga a fait du mariage de ses filles avec des empereurs une des priorités de sa politique. Nous allons maintenant tenter de comprendre comment il est parvenu à ses fins, en nous fondant sur ses notes personnelles.

\section{Des célébrations fastueuses}

Michinaga entourait de soins particuliers les femmes de sa famille qui étaient susceptibles de servir ses ambitions et qu'il considérait comme de potentielles futures épouses et mères d'empereur, à commencer par ses filles et sa première petite-fille, Teishi (1013-1094). Celle-ci fit l'objet d'une cérémonie particulièrement fastueuse en 1015 lorsqu'elle revêtit pour la première fois le pantalon. Michinaga a décrit cet événement en détail dans ses notes journalières :

Beau temps. Aujourd'hui, vêture du pantalon de la princesse Teishi. Je vais au palais [installé provisoirement en la résidence Biwa-dono des Fujiwara] vers midi. Vers quatre heures de l'après-midi, on dresse le dais michô, et on choisit cette heure parce qu'elle est faste. On a enlevé le dais qui était là, pour en dresser un nouveau, garni de tentures tobari en gaze usumono de couleur rouge sappan [appelée suô, Caesalpinia sappan $L$, proche du rouge bordeaux] dégradée vers le bas, et de [deux] miroirs suspendus, etc. Tout a été fabriqué de petite taille [car destiné à un enfant]. Il y a une paire de petites étagères zushi, une paire de coffrets à peignes kushibako, une paire de coffrets à cahiers zôshi-bako, une paire de coffrets à parfums kôkobako, un coffret-écritoire suzuri-bako et un brûle-parfum hitori. Tout cela est en laque d'or avec incrustations de nacre. L'accoudoir kyôsoku et le siège goza sont également de petite taille. Deux paires de paravents byôbu de trois pieds cinq pouces [d'un mètre de haut] et des écrans sur pied kichô, respectivement de trois pieds [90 cm] et de deux pieds cinq pouces $[75 \mathrm{~cm}]$.

6. Ôkagami, coll. Nihon Koten Bungaku Taikei, Tôkyô, vol. 21, 1960, p. 205-206; trad. H. Craig McCullough, Okagami, The Great Mirror : Fujiwara Michinaga (966-1027) and His Times. A Study and Translation, Princeton, 1980, p. 185, 186, 191. Le décompte de l'âge suit la tradition japonaise qui veut que l'enfant ait un an à sa naissance (et non pas zéro). Voir en fin d'article la liste des personnages mentionnés dans cet article. 
À sept heures du soir, la princesse prend place [...]. À l'heure prévue, vêture du pantalon : l'empereur [Sanjô, r. 1011-1016, son père], de sa main, noue les cordons sur les hanches de la princesse. Ensuite, on sert le repas de la princesse [...]. L'empereur se retire. [Après le repas de la princesse,] il paraît à nouveau pour le banquet des dignitaires [...]. [Pour le service de l'empereur,] on a fabriqué un [paysage miniature constitué] d'une montagne faite de bois d'aloès jin no yama, plantée d'arbres, [pour décorer le plateau impérial] avec six plats morimono. La table dai pour le saké est ornée d'un mont Hôrai, « montagne des immortels » [en miniature], et y sont disposés un pot tsubo et une coupe à saké sakazuki en verre [bleu lapis-lazuli] ruri ${ }^{7}$.

Le banquet continue avec un accompagnement musical et une deuxième ronde de coupes à saké. Il prend fin avec la distribution par Michinaga de costumes de soie en tant que gratifications, aux convives, parmi lesquels figuraient de nombreuses dames de cour venues rendre hommage à la petite princesse. Ce jour-là, le haut dignitaire Fujiwara no Sanesuke (957-1046), qui n'avait pourtant pas l'habitude de ménager Michinaga lorsqu'il s'agissait de critiquer les entorses au protocole des cérémonies, s'est montré, pour une fois, tout à fait admiratif. Il a estimé que les petits meubles en laque et le décor des plateaux impériaux étaient d'une élégance exceptionnelle et il avait tout particulièrement apprécié le « plateau à décor de bois d'aloès » et la « grève des immortels ${ }^{8} »$.

On peut en déduire que Michinaga avait porté une attention toute particulière au mobilier et au décor de la cérémonie de vêture du pantalon de sa petite-fille. De manière générale, les célébrations n'étaient pas censées refléter les goûts personnels de leurs commanditaires. Présidées directement ou indirectement par l'empereur, elles étaient en effet régies par les règles très strictes du protocole de la cour et considérées comme une charge officielle de la noblesse toute entière.

\section{Le choix des cérémonies comme méthode de gouvernement}

La vie de la cour japonaise était rythmée par les célébrations gyôji - c'est-à-dire des actes précis de caractère religieux ou profane, allant du rite à la procédure administrative, en passant par les événements sociaux, qui se succédaient tout au long de l'année. Ce système, d'inspiration chinoise, avait une fonction bien précise. Dans le Japon ancien et médiéval ( $\mathrm{VII}^{\mathrm{e}}-\mathrm{XVI}^{\mathrm{e}}$ siècles), les célébrations étaient régies par des rites rei qui étaient

7. Midô kanpakuki zenchûshaku, Chôwa 4.4.7. (1015): trad. F. HÉRAIL, Notes journalières..., vol. 3, p.95, 96. Teishi, née en Chôwa 2.7.7. (1013), a donc un an et huit mois. Voir F. HÉRAIL, La Cour du Japon à l'époque de Heian, aux Xe et XI siècles, Paris, 1995, «Les étapes de la vie », p. 141-142.

8. Shôyûki, Chôwa 4.4.7. 
censés avoir une influence sur les puissances supérieures en relation avec le principe $r i$, ordonnateur du monde. Elles avaient pour but de réguler les comportements humains et d'empêcher les désordres. Les rites conformes à l'ordre de l'univers auxquels ces cérémonies donnaient lieu étaient fixés par le cérémonial gi-shiki, composé de l'étiquette gi et du règlement shiki.

Le rôle de la cour impériale consistait à observer le déroulement annuel des célébrations afin que l'ordre de l'univers soit respecté et que l'harmonie règne dans le pays. Le souverain jouait le rôle d'intermédiaire entre les puissances supérieures et le pays, et il était considéré comme le bienfaiteur du peuple. Concrètement, ces bienfaits prenaient la forme de récompenses, de promotions ou de nominations dont faisaient l'objet les membres de son entourage.

Le cycle annuel des célébrations nenjûgyôji, et plus particulièrement celui des rites du culte shintô, a été consigné dans les premiers codes administratifs ritsuryô à partir du VII ${ }^{\mathrm{e}}$ siècle. On sait qu'en 885 , la résidence impériale Seiryôden comportait un ensemble de cloisons coulissantes sur lequel figurait le nenjûgyôji. D'après les traités de protocole, ce cycle se composait de quelque trois cents célébrations, dont une centaine étaient couramment pratiquées. Il y avait deux grands types de célébrations. Les unes étaient propres à l'administration de la cour, notamment les séances de nominations ou de proclamations, alors que les autres consistaient en des rites shintô ou bouddhiques. Les premières, d'ordre profane, avaient pour but d'exalter la bienveillance du souverain par des banquets et des événements d'ordre administratif qui mettaient en évidence la hiérarchie de la cour ou marquaient les étapes de la vie de ceux qui assuraient la continuité de la lignée impériale. Les secondes étaient adressées aux puissances supérieures de façon à écarter les calamités et les esprits malfaisants ou bien assurer la prospérité (en particulier en ce qui concerne les récoltes), le bonheur et la protection du pays. Elles se déroulaient à la cour et dans les résidences des nobles, conformément à un cérémonial très précis et les fautes concernant l'observation du protocole avaient la réputation d'être néfastes. Dans l'ensemble, les célébrations, loin d'être un divertissement, étaient considérées comme une tâche essentielle des nobles et des fonctionnaires de la cour. En fait, elles constituaient la charge primordiale du gouvernement ${ }^{9}$.

Mais, pour Fujiwara no Michinaga, ces célébrations étaient aussi l'occasion de mettre en évidence son rôle prééminent à la cour et sa position dominante dans la hiérarchie, notamment par la distribution de gratifications. Elles contribuaient également à souligner son prestige par le biais de décors fastueux. Et les ambitions du grand chancelier ne s'arrêtaient pas là. Il

9. Voir F. HéraIL, Notes journalières..., vol. 1, p. 50-107, «Cycle annuel des célébrations de la cour »; EAD., La Cour du Japon..., « Rites et célébrations », « Choix des rites comme méthode de gouvernement », p. 47-88. 
entendait en effet assurer par une habile politique matrimoniale une gloire encore plus grande à sa maison en la liant au pouvoir impérial. Pour illustrer notre propos, revenons un instant sur la cérémonie de la vêture du pantalon de la princesse Teishi.

\section{L'importance de l'apparat}

En 1015, la fabrication du décor de la cérémonie de vêture du pantalon de la princesse Teishi a occupé les artisans des ateliers attachés à la maison de Michinaga dès le début de l'année. Voici ce qu'on peut lire dans le Miroir de la splendeur des Fujiwara (Eiga monogatari) :

Les deux premiers mois se sont déroulés avec les célébrations habituelles. Mais cette année, la princesse allait avoir trois ans et la vêture du pantalon était prévue pour le quatrième mois. On a donc fait fabriquer des meubles miniatures dans les ateliers des Fabrications. La cérémonie devait avoir lieu à la résidence Biwa-dono, parce que c'est là que l'empereur résidait [à ce moment].

Enfin arriva le grand jour :

Monseigneur y a prêté une attention toute particulière et l'empereur a souhaité qu'on y apporte le plus grand soin possible. C'était un événement d'une splendeur au-delà de toute description et les festivités se sont poursuivies pendant trois jours ${ }^{10}$.

Michinaga a donc fait fabriquer spécialement pour l'occasion des pièces de mobilier dont l'aspect était conforme aux règles du « décor protocolaire » consignées dans les traités cérémoniels. L'un de ces traités, intitulé Notes relatives à divers usages essentiels, classés méthodiquement (Ruijû zatsuyôshô) (XII siècle), donne une description détaillée du décor de la résidence des régents Fujiwara. Pour chaque meuble, il indique ses mesures, ainsi que les matières premières et la main d'œuvre nécessaires, le tout étant illustré par des dessins. On y trouve également le décor mis en place lors de la célébration de la vêture du pantalon de la petite princesse Teishi.

10. Eiga monogatari, chap. 12, «Tama no muragiku», coll. Nihon Koten Bungaku Taikei, Tôkyô, vol. 1, 1961, p. 363, 364 ; trad. W. MCCullough et H. C. MCCullough, A Tale of Flowering Fortunes, Stanford, 1980 (2 vol.), vol. 2, p. 431, 432. Michinaga, initiateur de la séance, a fait fabriquer tout cela dans ses ateliers tsukumo dokoro (à l'origine le terme désignait « l'office des Fabrications » de la cour). Il financera également les fabrications pour une autre cérémonie (en 1019), cette fois pour son petit-fils, le prince Atsunaga : voir cidessous, n. 24. 
Le dais consistait en un cadre en forme de baldaquin soutenu par douze montants et orné de deux petits miroirs en argent et de ferrures en bronze doré. Les quatre faces de ce dais étaient pourvues de tentures en sergé de soie chinois agrémenté de pièces et de rubans teints en rouge sappan. Les deux petits miroirs et les ferrures dorées avaient coûté à eux seuls respectivement mille et plus de deux mille sept cents rouleaux de taffetas de soie. La teinture rouge sappan et les soieries étaient importées de Chine ${ }^{11}$. Le sol était quant à lui recouvert de tatamis à bordures de brocart où étaient disposés des sièges ronds et un coussin en brocart chinois.

Le mobilier comprenait de nombreux coffrets en laque d'or avec incrustations de nacre. Les frais engagés pour chacun d'entre eux incluaient la main-d'œuvre et les matières premières. Selon le Ruijû zatsuyôshô, mentionné plus haut, qui évalue leur coût en rouleaux de taffetas de soie, pour réaliser chaque coffret à parfums laqué, il a fallu payer soixante-dix rouleaux de soie pour le travail d'ébénisterie, mille trois cents pour acheter la nacre et deux cents pour les travaux de ciselage et d'incrustation. Les laqueurs ont reçu cinq cent soixante rouleaux de soie pour le travail de la laque d'or, y compris le polissage, et ils ont utilisé 1,3 shô (un litre) de laque et vingt-neuf onces ( $400 \mathrm{~g}$ ) d'or. Soit au total plus de deux mille rouleaux de soie pour fabriquer un seul coffret à parfums. Les artisans qui travaillaient dans les ateliers des grands personnages étaient non pas salariés, mais rémunérés à la tâche et on leur fournissait les matières premières. Les deux coffrets de la princesse Teishi contenaient chacun des encens et des parfums, disposés dans quatre petits pots en argent. La nacre destinée aux incrustations sur laque provenait des lointaines îles du Sud (l'Okinawa actuel) et les fournitures des coffrets comprenaient presque exclusivement des denrées exotiques importées de Chine et provenant de pays situés sur les routes de la soie ${ }^{12}$. Comme les brocarts et les sergés de soie chinois,

11. Ruijû zatsuyôshô, coll. Gunsho ruijû, vol.26, Tôkyô, 1991, p. 590-596, section « chô [dais]»; KAWAMOTO S., KoIzUMI K. éd., Ruiju zatsuyôshô sashizukan, Tôkyô, 1998, p. 67-71, 195-200, 252, 253 ; AKIYAMA K. et al. éd., Genji monogatari zuten, Tôkyô, 1997, p. 51. Les soieries et le sappan faisaient partie des importations de Michinaga (voir C. voN VERSCHUER, Le Commerce entre le Japon, la Chine et la Corée à l'époque médiévale, VII'XVI siècles, Paris, 2014, p. 37-38, 68-69).

12. Ruijû zatsuyôshô, p. 577, section «kôko-bako»; KAWAMOTO S., KoIzUmI K. éd., Ruiju zatsuyôshô sashizukan, p. 60, 189 ; AKIYAMA K. et al. éd., Genji monogatari zuten, p. 52. Selon cette source, il s'agit de bois d'aloès jinkô, de girofles chôji, de valériane kanshôkô, de boswellia kunrokukô, de storax sogô, de cannelle keishin, d'agastache kawamidori (la seule denrée japonaise, une labiatée comme le shiso), et de musc jakô. Tous ces encens, ainsi que les soies chinoises, étaient importés du continent par Michinaga : voir C. VON VERSCHUER, Le Commerce entre le Japon ..., p. 65-68, 205-206. Lors de la cérémonie de 1015 (Midô kanpakuki, Chôwa 4.4.7.), le mobilier protocolaire était le suivant : dais michô, tentures tobari, teinture sappan suô, sergé chinois kara aya, brocart chinois kara nishiki, étagère zushi, coffret à peignes kushibako, coffret à cahiers yôshibako (ou zôshibako), coffret à parfums kôkobako, coffret-écritoire suzuribako, tous en laque d'or avec incrustations de 
les aromates les plus recherchés étaient de provenance étrangère. Autour $\mathrm{du} \mathrm{XI}^{\mathrm{e}}$ siècle, l'empereur et les hauts dignitaires ont importé à grands frais ces précieuses denrées du continent asiatique. Pour la noblesse de Heian, les décors prestigieux, les costumes somptueux et les encens exotiques faisaient partie intégrante de la vie de cour.

Le Roman du Genji fait allusion à des concours de parfums où le prince Genji jouait le rôle d'arbitre. Par exemple, pour le (parfum) jijû, le prince choisit celui du ministre : «C'est un parfum de haute qualité, séduisant et évocateur!», dit-il. Ceux de la dame de l'aile orientale (la dame Murasaki, l'épouse principale du Genji) étaient de trois sortes, parmi lesquelles la Fleur de prunier se distinguait par sa vivacité au goût du jour, relevée d'une pointe d'exubérance qui lui donnait une fragrance des plus rares. «Assorti à la brise de cette saison, nul parfum ne surpasserait celuici ! », estima le prince ${ }^{13}$.

La princesse Teishi devait disposer d'un assortiment de parfums dès son plus jeune âge. Les essences exotiques faisaient en effet partie de l'apparat qui allait de pair avec la vie privée et publique de l'aristocratie. Et il en allait de même pour les soies importées.

Parmi les soies chinoises les plus prisées, il y avait le sergé dont on faisait des vêtements de l'aristocratie, et le brocart utilisé le plus souvent pour l'ameublement du palais et des résidences nobles. Voici ce qu'on peut lire dans le Roman $d u$ Genji à propos des préparatifs ordonnés par l'empereur retiré pour la cérémonie de la vêture de la traîne ${ }^{14}$ :

La décoration du lieu de la cérémonie, une salle exposée à l'ouest du Pavillon du Chêne, à commencer par le dais et les tentures, ne comportait aucun sergé ni brocart de notre pays, si bien que la salle évoquait les parures des princesses de Chine, tant le souverain l'avait voulue magnifique, imposante, en un mot éclatante [...] Le souverain avait à cette occasion offert un grand nombre d'objets de Chine provenant des magasins de sa chancellerie privée ${ }^{15}$.

nacre makie raden; accoudoir kyôsoku, brûle-parfum hitori, paravent byôbu, écran sur pied kichô. Le Ruijû zatsuyôshô décrit leur fabrication en détail, p. 575-609.

13. Genji monogatari, livre Umegae, coll. Nihon Koten Bungaku Taikei, vol. 3, Tôkyô, 1971, p. 164-165 ; trad. R. SiEfFerT, MuRASAKI ShIKIBU, Le Dit du Genji, Paris, 1985, vol. 1, p. 602 (la traduction est légèrement modifiée).

14. Cérémonie qui marque le passage à la majorité des jeunes filles.

15. Genji monogatari, livre Wakana jô, coll. Nihon Koten Bungaku Taikei, vol. 3, p. 231 ; trad. R. SiefFert, MurasaKi ShiKibu, Le Dit du Genji, vol. 2, p. 20. Pour les décors de brocart nishiki et de sergé aya, voir aussi le Livre Umegae (coll. Nihon Koten Bungaku Taikei, vol. 3, p. 159 sq.). Voir encore KAWAZOE F., Karamono no bunkashi, Tôkyô, 2014, p. 63-94. 
Le coût total de la fabrication du décor et des dons destinés à la célébration de la vêture du pantalon de la princesse Teishi de 1015 se chiffrait en milliers de rouleaux de taffetas de soie, ce qui constitue une somme énorme si on la compare, par exemple, au prix du terrain d'une résidence à la capitale de Heian ${ }^{16}$, et ce uniquement pour les frais de main-d'œuvre et les matières premières. À l'époque, on fabriquait dans toutes les provinces du Japon du taffetas de soie, c'est-à-dire de la soie à armure simple. Ce taffetas était ensuite livré en guise de taxes à la cour impériale. Le grand chancelier devait disposer de quantités importantes de cette étoffe, car il en a distribué tout au long de sa carrière sous forme de rouleaux ou de vêtements de soie en guise de gratifications pour des services rendus. Fujiwara no Michinaga ne reculait devant aucune dépense pour se procurer des objets de prestige, aussi bien japonais qu'étrangers. Il payait en pépites d'or les commerçants chinois qui lui faisaient parvenir des soies précieuses, des parfums et des remèdes exotiques. Ses ressources lui permettaient d'acquérir ces denrées précieuses, très recherchées. Voyons maintenant en quoi consistaient les revenus et la fortune du premier personnage de la cour.

\section{La mainmise de Michinaga sur les richesses du pays}

Les terres du pays tout entier passent sous le contrôle d'une seule famille ; ce qui reste sous celui de la cour, n'est-ce pas seulement la pointe d'un foret? En quel siècle vivons-nous ${ }^{17}$ !

C'est en ces termes que le dignitaire de la cour Fujiwara no Sanesuke exprime son mécontentement à l'égard de Michinaga. Selon lui, le grand chancelier s'enrichissait aux dépens des impôts qui étaient normalement versés à la cour impériale. Mais l'accaparement des « terres du pays tout entier » dénoncé par Sanesuke ne constituait qu'une partie de la stratégie de mainmise du grand chancelier sur les richesses du pays.

Ses revenus étaient d'origines diverses. Bien que les sources soient lacunaires à ce sujet, on pense qu'ils se composaient de dons, de revenus fiscaux, de revenus domaniaux et de redevances.

Le grand chancelier recevait des dons sous forme de cadeaux quasiment obligatoires qui représentaient une partie des revenus des fonctionnaires de la cour assignés au service dans sa maison, les keishi (sans

16. Heian ibun, éd. TAKeUCHI R., Tôkyô, vol. 7, 1965, p. 9. En 1170, un certain Ki no Suemasa a vendu un petit terrain de $300 \mathrm{~m}^{2}$ situé dans la partie sud de la ville, sur la Huitième Avenue, pour 11 rouleaux de taffetas de soie. On peut donc en déduire qu'un terrain de $3000 \mathrm{~m}^{2}$ valait à peu près 110 rouleaux de taffetas de soie. Michinaga a dépensé des milliers de rouleaux de soie pour une seule célébration.

17. Shôyûki, Manju 2.7.11.(1025). 
doute une vingtaine de personnes). Il recevait également des dons de la part des personnes attachées à sa maison par des liens de clientèle, les kenin (dont le nombre exact n'est pas connu) ${ }^{18}$. Les banquets et autres fêtes qui se déroulaient chez Michinaga étaient financés par ces hommes. Lors de ces fêtes, il leur remettait des «cadeaux » qui étaient des produits qu'il avait reçus auparavant de leur part. De même, les fonctionnaires qui cherchaient à obtenir un poste de gouverneur offraient des chevaux à Michinaga. Une fois nommés, les gouverneurs finançaient en retour la construction de résidences ou de temples patronnés par le grand chancelier.

Par ailleurs, la moitié, voire davantage, des produits fournis par les provinces au titre de l'impôt public finissait dans les greniers de Michinaga. Le reste des revenus fiscaux, qui devait revenir théoriquement à la cour, c'est-à-dire dans les magasins des bureaux de l'administration, était en fait accaparé par les empereurs ou certains dignitaires. Michinaga, pour sa part, redistribuait ensuite ces recettes sous forme de gratifications aux fonctionnaires ou d'aumônes aux moines, ce qui lui permettait d'entretenir des liens de clientèle.

Le grand chancelier percevait aussi des revenus domaniaux familiaux. Il disposait en effet de domaines shôen attachés à la charge de chef de la maison Fujiwara qu'il a occupée durant sa vie, mais on ne dispose que de peu d'informations à ce sujet.

Michinaga devait également percevoir des revenus domaniaux personnels même si rien ne permet d'affirmer que des domaines lui aient appartenu à titre personnel. En effet, les titres de propriété et les exemptions ne sont devenus courants que plus tard, à l'époque de Fujiwara no Yorimichi (992-1074), son fils. On peut supposer que les hommes avec lesquels Michinaga entretenait des liens de clientèle versaient une partie des revenus provenant de leurs propres domaines, autrement dit des taxes qu'ils devaient au titre de l'impôt, non pas au siège des provinces, mais au grand chancelier, et que les gouverneurs (notamment ceux de sa clientèle) toléraient ces « exemptions ».

Le grand chancelier percevait par ailleurs des redevances constituées par la moitié de la taxe foncière en riz payée par plusieurs milliers de foyers concédés fuko qui lui avaient été attribués à titre de rente. Il accaparait aussi

18. Les keishi, fonctionnaires supérieurs attachés à la maison d'un haut dignitaire, consacraient une partie de leurs activités aux affaires de cette maison. Les kenin n'étaient pas officiellement attachés comme fonctionnaires à une maison de dignitaire, mais plutôt à un haut dignitaire au titre d'un lien de clientèle. Ces personnes achetaient en quelque sorte la protection de Michinaga par d'importants cadeaux. Par exemple, ce dernier pouvait faire nommer un kenin à un poste de gouverneur de province et attendre en contrepartie des livraisons importantes de biens de ladite province. Voir F. HÉRAIL, La Cour et l'administration du Japon à l'époque de Heian, Genève, 2006 (rééd.), p. 527-530 ; EAD., Notes journalières..., vol. 1, p. 23, 590, vol. 3, p. 380. 
les recettes des foyers concédés à ses fils en tant que hauts dignitaires, et aux impératrices, ses filles.

En fait, toutes ces ressources ne constituaient ni plus ni moins qu'un détournement de redevances domaniales et de taxes publiques. Michinaga se considérait comme un personnage public qui représentait la cour et l'empereur. À ce titre, il se permettait de faire transiter par sa maison une part importante des richesses du pays qu'il redistribuait ensuite à sa clientèle de dignitaires et de fonctionnaires ou qu'il utilisait au profit de la maison impériale. Ce faisant, il a contribué au déclin de l'administration de la cour et des provinces selon le système bureaucratique qui avait été institué par les codes au début du VIII ${ }^{\mathrm{e}}$ siècle ${ }^{19}$.

\section{Le déclin du système fiscal instauré par les codes}

Au fil du temps, le système fiscal japonais avait évolué vers une structure propice au développement des liens de clientèle. Au VIII ${ }^{\mathrm{e}}$ siècle, les soixante-huit provinces du Japon livraient annuellement un volume fixe de produits à la capitale au titre de l'impôt, la nature des produits étant définie par le code administratif ritsuryô de 701. Ces impôts étaient de trois sortes : des taxes en riz, des taxes en nature (denrées alimentaires, matières premières, produits artisanaux) et des corvées (en qualité de gardes à la cour, de soldats dans les provinces ou de main-d'œuvre pour la construction d'édifices publics). Quant aux gouverneurs de province c'étaient des fonctionnaires rémunérés par la cour impériale, nommés pour quatre ans.

Aux IX ${ }^{\mathrm{e}}$ et $\mathrm{X}^{\mathrm{e}}$ siècles, les arriérés et les impayés se sont multipliés. Le recensement de la population et le cadastrage des terres ont progressivement cessé et les quotas des fournitures fiscales n'ont plus été respectés. Le volume fixe des livraisons annuelles dues par chacune des provinces a peu à peu laissé place à un système de commandes formulées par la cour impériale en fonction de ses besoins et réparties de façon inégale entre les provinces. L'approvisionnement était toujours géré par l'appareil administratif dans le respect de la hiérarchie des services de la cour. Mais des liens de clientèle se sont constitués entre les gouverneurs de province et les dignitaires de la cour, entraînant la modification du statut de gouverneur de province. Ayant cessé d'être rémunérés par la cour, les gouverneurs ont été amenés à tirer leur revenu directement de la province dont ils avaient la charge. Dès lors, ils se sont comportés davantage en hommes d'affaires qu'en fonctionnaires. La levée de l'impôt a été de plus en plus laissée à leur discrétion, tandis qu'ils s'engageaient personnellement auprès des dignitaires pour les livraisons qu'ils devaient faire à la cour. Les hauts dignitaires ont peu à peu pris le contrôle de la distribution des biens livrés par les provinces aux bureaux de

19. F. Hérail, Notes journalières..., vol. 1, p. 13, 14. 
l'administration. Et c'est ainsi que les liens de clientèle ont joué un rôle de plus en plus important.

À partir du XI ${ }^{\mathrm{e}}$ siècle, l'approvisionnement de la cour n'a plus suivi la voie hiérarchique et bureaucratique. Les ministres se sont occupés personnellement de cette tâche en traitant directement avec les gouverneurs. Depuis le $\mathrm{X}^{\mathrm{e}}$ siècle, les gouverneurs de province avaient commencé à aménager de façon illégale des greniers privés dans leur résidence de la capitale pour engranger les recettes publiques qui leur étaient adressées et, au XI ${ }^{\mathrm{e}}$ siècle, ces greniers ont fini par être officiellement tolérés par les grands de la cour, afin de soutenir les finances publiques ${ }^{20}$. Cette évolution a contribué à l'afflux des biens provinciaux vers les greniers de Michinaga.

\section{Une immense fortune personnelle}

Mais Fujiwara no Michinaga disposait d'autres ressources que les revenus fiscaux et fonciers dont il vient d'être question. Nous pouvons nous faire une idée du contenu de ses magasins grâce à un incident qui se produisit en 1017. Des voleurs s'introduisirent dans la résidence du grand chancelier et emportèrent deux mille onces $(28 \mathrm{~kg})$ d'or et huit cents onces $(11,2 \mathrm{~kg})$ d'argent. Une enquête fut menée et sept policiers, qui furent récompensés pour l'occasion, retrouvèrent mille onces d'or et deux cents onces d'argent. Un suspect fut convoqué, puis relâché, avant que les voleurs, au nombre de trois, ne soient finalement arrêtés. Les trois hommes étaient tous des suivants de maisons de fonctionnaires moyens et ils déclarèrent avoir revendu le reste de l'or dérobé, ainsi que des étoffes précieuses de Chine ${ }^{21}$.

À l'époque, l'or était utilisé non seulement pour acheter des produits importés de Chine, mais aussi pour fabriquer des objets d'artisanat de prestige, notamment de la vaisselle en bronze doré et des meubles en laque d'or. Il provenait des gisements fluviaux de la province de Mutsu, en particulier ceux de la région de Fukushima, d'où il était extrait par le procédé du lavage qui permettait de débarrasser les pépites de leur gangue ${ }^{22}$.

Michinaga avait donc accumulé une quantité d'or considérable dans ses magasins. Ceux-ci contenaient aussi des soieries chinoises, ainsi que de nombreux parfums et remèdes exotiques qu'il avait importés de Chine. La fortune du grand chancelier était du même ordre que celle d'un personnage

20. FURUSE N., Sekkan seiji, Tôkyô, 2011, p. 46, 134-146.

21. Midô kanpakuki, Kannin 1.5.27., et 1.7.10. à 12. (1017): F. HÉRAIL, Notes journalières..., vol. 3, p. 368, 379-381; Shôyûki, Kannin 1.7.10. et 16. Voir G. C. HuRST, «Kugyô and Zuryô, Center and Periphery in the Era of Fujiwara no Michinaga », dans M. Adolphson et al. éd., Heian Japan, Centers and Peripheries, Honolulu, 2007, p. 66-101 (ici p. 76).

22. Voir C. von VerschUER, Le Commerce..., p. 44-52, pour les importations payées en or. 
richissime appelé Nakayoshi, dont on sait qu'un siècle après Michinaga, il avait entassé « sept jarres remplies d'or, soixante-dix mille onces d'argent et, en outre, d'innombrables produits exotiques [karamono] et objets de prestige japonais [wamono] » dans les greniers de sa résidence de Heian ${ }^{23}$.

Aux XI ${ }^{\mathrm{e}}-\mathrm{XII}^{\mathrm{e}}$ siècles, les Japonais les plus fortunés possédaient de l'or et de l'argent, des produits importés de Chine et des objets de luxe japonais comme des meubles en laque d'or incrustés de nacre. L'or était étroitement lié à l'apparat. En 1019, Michinaga fournit 35 onces (491 g) d'or pour faire fabriquer deux étagères zushi, ainsi que l'étui d'une coiffe de cour pour la célébration de la majorité du prince héritier, son petit-fils Atsunaga, le futur empereur Gosuzaku. L'or était parfois difficile à se procurer même pour les hauts dignitaires. Ainsi, Fujiwara no Sanesuke dut renoncer à la laque d'or pour certaines pièces du trousseau ${ }^{24}$ qu'il fit fabriquer pour le mariage de sa fille en 1023. Celui-ci était composé du même mobilier que celui de la princesse Teishi, évoqué plus haut, mais le haut dignitaire ne réussit pas à se procurer la quantité d'or nécessaire. La grande richesse de Michinaga lui permit d'organiser des célébrations somptueuses qui ont fait sa gloire, et en particulier celles destinées à ses héritiers.

\section{Un décor somptueuxà la mesure de la gloire de son commanditaire}

En 1008, on célébra ainsi avec faste le centième jour momoka depuis la naissance du jeune prince Atsuhira, premier fils de Shôshi, la première fille du grand chancelier. Tous les dignitaires de la cour étaient présents. À cette occasion, dix d'entre eux offrirent conjointement à l'enfant cent paniers remplis de fruits et de gâteaux. Ces paniers étaient tressés en fils d'or et d'argent et rehaussés d'un décor de perles de verre. Les fonctionnaires de la nouvelle maison du jeune prince lui présentèrent cent coffres en bois ployé, « objets admirables, dont la splendeur ne peut se décrire ». Pour le banquet auquel cette célébration a donné lieu, les plateaux de chaque convive étaient en argent et celui de l'empereur Ichijô - à la fois père du prince, neveu et gendre de Michinaga - comportaient une représentation de la «grève des immortels » $(\text { suhama })^{25}$. Le plat de riz cuit réservé au

23. Chôshûki (Notes journalières de Minamoto no Morotoki ([1077-1136]), Chôshô 3.5.2. (1134); Chôshûki, coll. Zôhô Shiryô Taisei, éd. SHIRYô TAISEI KANKÔKAI, vol. 16 et 17, Kyôto, 1965 ; G. C. HuRST, « Kugyô and Zuryô... », p. 76. Nakayoshi, dont le nom de famille est inconnu, était un ancien gouverneur de Chikugo ; il possédait des magasins jouxtant le temple Ninnaji à Kyôto et, à un certain moment, gérait les biens de la princesse consacrée du sanctuaire de Kamo ; voir TODA Y., Shoki chûsei shakai-shi no kenkyû, Tôkyô, 1991, p. 214.

24. Midô kanpakuki, Kannin 3.3.2. (1019) : F. HÉRAIL, Notes journalières..., vol.3, p. 602 ; Shôyuki, Jian 3.6.17. (1023).

25. Sur ce décor, voir, dans ce numéro de Médiévales, l'article de Michel VieillardBaron, « Les concours de poèmes comme rituels de cour. Autour du "Concours de poèmes 
souverain était découpé en parts formant une tortue - symbole de longévité par excellence - dont la carapace était surmontée par le mont Hôrai, où résident des immortels. «Et ce n'était pas tout», ajoute Michinaga. Les mets d'accompagnement du souverain étaient disposés sur un autre plateau, avec un paysage figurant une étendue d'eau, des oiseaux et des rochers. Il y avait aussi une table avec des pieds en forme de pattes de héron sur laquelle était posé le service à saké de l'empereur constitué d'une coupe et d'une aiguière en verre bleu lapis-lazuli ${ }^{26}$.

Dans son journal, Michinaga se livre à quatre reprises à la description détaillée de ces décors fastueux, révélant son extrême attention à ces cérémonies : deux fois pour son premier petit-fils Atsuhira - en 1008, pour la cérémonie du centième jour, et en 1010, pour celle de la vêture du pantalonet deux fois pour sa première petite-fille Teishi, pour les mêmes célébrations en 1013 et en $1015^{27}$. La famille impériale, qui était directement liée à Michinaga par les mariages et les liens du sang, assistait à ces célébrations et les hauts dignitaires ne pouvaient pas manquer de constater les progrès de la mainmise du grand chancelier sur la cour. Le principe de politique matrimoniale en soi était en fait une pratique banale très répandue parmi la noblesse de cour. Mais le grand chancelier a soutenu sa politique par des investissements importants consacrés au protocole de cour. Il a mis au jour une habileté particulière pour faire briller sa descendance sous les yeux d'une nombreuse assistance. Le faste du décor des cérémonies a souligné devant tous la prééminence de sa lignée.

Michinaga atteignit le sommet de sa gloire en 1018, quand sa troisième fille Ishi devint impératrice et c'est à cette occasion qu'il composa le poème mentionné au début de cet article. Ce jour-là marquait l'aboutissement des célébrations qui avaient précédé les noces impériales. Une fois arrivé au sommet de sa gloire, Fujiwara no Michinaga, qui n'avait pourtant pas coutume d'exprimer ses sentiments dans ses notes journalières, fit une exception à l'occasion des noces de sa fille Ishi avec l'empereur Goichijô, en 1018. Quatre jours après le banquet organisé à cette occasion, il écrivit en effet ce qui suit à propos d'une réunion familiale :

tenu au palais impérial la quatrième année de l'ère Tentoku [960]" (Tentoku yonen dairi uta awase) ».

26. Midô kanpakuki zenchûshaku, Kankô 5.12.20. (1008): F. HÉRAIL, Notes journalières..., vol.2, p. 287-288 ; Shôyûki, Kankô 5.12.20.; Gonki (Notes journalières de Fujiwara no Yukinari [972-1027]), Kankô 5.12.20 ; Gonki, coll. Zôho Shiryô Taisei, éd. SHIRYÔ TAISEI KANKÔKAI, vol. 4 et 5, Kyôto, 1965.

27. Midô kanpakuki zenchûshaku, Kankô 7.10.22. (1010): trad. F. HÉRAIL, Notes journalières..., vol. 2, p. 434-435 ; Midô kanpakuki, Chôwa 2.10.20. (1013) : trad.F. HéRAIL, Notes journalières..., vol. 3, 50-51. Voir plus haut, n. 7, pour la vêture du pantalon de Teishi en 1015. 
Il y a l'entrevue des trois impératrices. C'est un spectacle qui fait naître une joie sans bornes. La jeune princesse [Teishi] est aussi présente, ainsi que la mère des impératrices [Rinshi] et ma fille du troisième rang [Kishi]. Je suis tout éperdu de voir une telle chose de mon vivant. Impossible d'exprimer complètement ce que je ressens. C'est une chose comme on n'en a jamais vue $^{28}$.

Les célébrations représentaient une préoccupation majeure pour Fujiwara no Michinaga comme pour tous les dignitaires de la cour, car elles constituaient la tâche primordiale des courtisans. Elles étaient en effet censées contribuer au maintien de l'ordre et de l'harmonie dans le pays. Mais le grand chancelier s'en est aussi servi en outre pour instrumentaliser sa politique de mariage. Il mit à contribution le protocole de cour pour mettre en scène des cérémonies d'un éclat hors du commun. Il impressionna son entourage et imposa toujours plus la prééminence de ses héritiers. L'avenir de sa lignée lui tenait plus à cœur que la gestion des affaires administratives. Sa fortune considérable lui permettait d'organiser des séances d'une telle somptuosité que les membres de son entourage en étaient éblouis. L'apparat jouait un rôle clé dans la réussite de ces événements, soulignant la position prééminente du grand chancelier et sa mainmise sur les affaires du pays.

Sa fortune, le chancelier l'a construite grâce à stratégie d'appropriation des richesses du pays au profit des greniers de sa propre maison. Michinaga mit à contribution sa clientèle provinciale pour recueillir dans ses propres greniers une part importante des richesses du pays. Sous son influence, la gestion bureaucratique des finances publiques passa aux mains des dignitaires de la cour et évolua vers un fonctionnement clientéliste. Le chancelier put ainsi entretenir une clientèle de plus en plus nombreuse parmi les dignitaires. Les mécanismes du pouvoir en ont été modifiés. Sous l'impulsion de Michinaga, la gestion administrative du pays a connu une mutation qui fut à l'origine d'une nouvelle ère politique dans le Japon de l'époque de Heian.

Charlotte von Verschuer - École Pratique des Hautes Études (Paris)

28. Midô kanpakuki zenchûshaku, Kannin 2.10.22. (1018): trad. F. HÉRAIL, Notes journalières..., vol. 3, p. 549. 


\section{Personnages mentionnés dans l'article :}

- Fujiwara no Michinaga (966-1027), 996, ministre de gauche ; 1017, ministre des affaires suprêmes ; 1018, se retire; 1019, devient moine ; dans cet article, on l'appellera aussi « le grand ministre .

- L'empereur Ichijô (980-1011, r. 986-1011), neveu et gendre de Michinaga, fils de l'empereur En.yû.

- L'empereur Sanjô (976-1017, r. 1011-1016), neveu et gendre de Michinaga, fils de l'empereur Reizei.

- L'empereur Goichijô (1008-1036, r. 1016-1036), Atsuhira, petit-fils de Michinaga, premier fils de sa première fille Shôshi.

- L'empereur Gosuzaku (1009-1045, r. 1036-1045), Atsunaga, petit-fils de Michinaga, deuxième fils de Shôshi.

- L'empereur Goreizei (1025-1068, r. 1045-1068), Chikahito, arrière-petit-fils de Michinaga, fils de Gosuzaku et Kishi.

- L'empereur Gosanjô (1034-1073, r. 1068-1072), Takahito, arrière-petit-fils de Michinaga, fils de Gosuzaku et Teishi.

- Fujiwara no Shôshi (988-1074), $1^{\text {re }}$ fille de Michinaga, $2^{\mathrm{e}}$ épouse de l'empereur Ichijô, mâ̂tresse de Murasaki Shikibu.

- Fujiwara no Kenshi (994-1027), $2^{\mathrm{e}}$ fille de Michinaga, épouse de l'empereur Sanjô.

- Fujiwara no Ishi (999-1036), 3e fille de Michinaga, épouse de l'empereur Goichijô.

- Fujiwara no Teishi (1013-1094), petite-fille de Michinaga, épouse de l'empereur Gosuzaku.

Le ministre Fujiwara no Michinaga (966-1027) : politique matrimoniale, apparat et liens de clientèle à l'époque du Roman du Genji

Fujiwara no Michinaga, premier personnage de la cour à l'époque du Roman du Genji, mit en œuvre une politique matrimoniale qui a assuré à sa famille le pouvoir impérial pour trois générations. II mit en scène des cérémonies qui exaltent son prestige et employait sa clientèle pour s'assurer les ressources financières nécessaires pour réaliser ses ambitions. II en résulta une mutation de la structure du pouvoir pour les générations à venir.

Japon - cour impériale - clientèle - cérémonial - prestige - pouvoir

The Minister Fujiwara no Michinaga (966-1027) : Matrimonial Politics, Pageantry and Patronage at the Time of the Tale of Genji.

Fujiwara no Michinaga, first political power at the Heian court at the time of Tale of Genji, implemented his matrimonial strategy to secure to his family the political power over three generations, organized the court ceremonies in a way to exalt his glory, and made use of a large clientele to secure for himself the necessary revenues to implement his policies. As a result the structure of power was modified for the future generations.

Japan - Imperial Court - Pageantry - Ceremonial - Prestige - Power 
